\title{
P-108
}

\section{Stilbenoid Compounds from Rhizomes of Smilax Myosotiflora}

\author{
Aboubaker A. Elhadi*, Hasnah Osman and K. C. Wong \\ School of Chemical Sciences, Universiti Sains Malaysia, Penang 11800, Malaysia; E-mail: aboubaker1971@yahoo.com
}

Two stilbenoid compounds, trans-3,4',5-trihydroxystilbene (1), and trans-3,3',5,5'-tetrahydroxy-4'-methoxystilbene (2) were isolated from ethyl acetate extract of the rhizomes of Smilax myosotiflora by using silica gel and sephadex LH-20 column chromatography. Their structures were characterized on the basis of MS, 1D and 2D NMR spectroscopic analysis and confirmed by spectral data available in the literatures. These compounds were the first time isolated from the rhizomes of Smilax myosotiflora.

Keywords: Smilax myosotiflora, rhizomes, ethyl acetate extract, column chromatography, NMR and mass spectroscopy. 